Article

\title{
Metabolic Profiling of Lactococcus lactis Under Different Culture Conditions
}

\author{
Kamalrul Azlan Azizan, Syarul Nataqain Baharum * and Normah Mohd Noor \\ Institute of Systems Biology, Universiti Kebangsaan Malaysia, 43600 Bangi, Selangor, Malaysia \\ * Author to whom correspondence should be addressed; E-Mail: nataqain@ukm.my; \\ Tel.: +603-8921-4550; Fax: +603-8921-3398.
}

Received: 25 May 2012; in revised form: 13 June 2012 / Accepted: 14 June 2012 /

Published: 3 July 2012

\begin{abstract}
Gas chromatography mass spectrometry (GC-MS) and headspace gas chromatography mass spectrometry (HS/GC-MS) were used to study metabolites produced by Lactococcus lactis subsp. cremoris MG1363 grown at a temperature of $30^{\circ} \mathrm{C}$ with and without agitation at $150 \mathrm{rpm}$, and at $37{ }^{\circ} \mathrm{C}$ without agitation. It was observed that L. lactis produced more organic acids under agitation. Primary alcohols, aldehydes, ketones and polyols were identified as the corresponding trimethylsilyl (TMS) derivatives, whereas amino acids and organic acids, including fatty acids, were detected through methyl chloroformate derivatization. HS analysis indicated that branched-chain methyl aldehydes, including 2-methylbutanal, 3-methylbutanal, and 2-methylpropanal are degdradation products of isoleucine, leucine or valine. Multivariate analysis (MVA) using partial least squares discriminant analysis (PLS-DA) revealed the major differences between treatments were due to changes of amino acids and fermentation products.
\end{abstract}

Keywords: Lactococcus lactis subsp. cremoris; metabolic profiling; GC-MS; derivatization

\section{Introduction}

Lactococcus lactis is a facultative anaerobe widely used for dairy production and food fermentation [1,2]. As a member of lactic acid bacteria (LAB), this bacterium typically produces lactate as a major fermentation end product causing medium acidification. The acidification process by L. lactis is important as the low $\mathrm{pH}$ not only inhibits growth of other microorganism, but also contributes to changes in food texture and biochemical conversions that produce flavors and aromas [3]. 
The growth of L. lactis is strongly influenced by different of stresses, including acidity, temperature and starvation of carbon or nitrogen. As an example, coagulation steps by acidification and heating during cheese manufacturing process affects the growth of L. lactis which enhance metabolite changes that influence the texture, taste and smell of the produced cheese [3-6].

L. lactis is auxotrophic for specific amino acids which are also involved in its adaptation to environmental stress $[7,8]$. Amino acids also make a significant contribution towards the production of metabolites that are associated with flavor and aromatic properties [9]. The relationship between the fermentation end products and changes of amino acids under the influence of temperature and agitation are of great interest because it relates to the central carbon metabolism [10], for better understanding of bacteria adaptation to environmental stress.

In order to assess the response to environmental stresses, a quantitative and qualitative study of L. lactis was carried out using gas chromatography-mass spectrometry (GC-MS) and headspace (HS) analysis. GC-MS requires metabolites to be volatile through derivatization. Common derivatization reagents are trimethylsilyl- (TMS) and methylchloroformate- (MCF) [11]. TMS is effective for sugars, polyols and sulfur-containing compounds, while MCF is preferable for amino acids [12]. TMS allows good fragmentation during electron impact, but the preparation usually requires a long heating step [13]. The application of rapid microwave-assisted (MA) technique in TMS derivatization procedure successfully shortened the heating process [13]. Unlike TMS, which requires completely dry samples, MCF derivatization allows direct derivatization of samples in aqueous solution without heating [11].

The aim of this research is to evaluate and determine the extracellular metabolic changes produced by L. lactis in response to temperature and agitation. Different derivatization agents was applied to analyze a wide range of metabolites The results were then compared and analyzed using multivariate analysis (MVA) of hierarchical clustering analysis and partial least squares discriminant analysis (PLS-DA).

\section{Results and Discussion}

\subsection{Homolactic and Mixed-Acid Fermentation}

Primary alcohols, aldehydes, ketones, polyol and organic acids of carboxylic acids were present in extracts of L. lactis grown at a temperature of $30{ }^{\circ} \mathrm{C}$ with and without agitation at $150 \mathrm{rpm}$, and at $37{ }^{\circ} \mathrm{C}$ without agitation (Table 1). Principal component analysis (PCA) revealed that the metabolic profiles at $30^{\circ} \mathrm{C}$ and $37{ }^{\circ} \mathrm{C}$ without agitation were different (Figure 1). PLS-DA derived loading plot analysis was used to identify metabolites that are significant for the differences (Figure 2). The first two principal components plots showed that fermentation end products of lactate, ethanol and acetate, followed by several organic acids belonging to the tricarboxylic acid (TCA) cycle are detected. Lactate, the major fermentation product of $\mathrm{LAB}[1]$, contributed the most under $37^{\circ} \mathrm{C}$ growth conditions, compared to ethanol and acetate at $30^{\circ} \mathrm{C}$ without agitation. This indicates a shift in metabolism from mixed-acid fermentation at $30^{\circ} \mathrm{C}$ to homolactic fermentation at $37^{\circ} \mathrm{C}$. 
Table 1. Detected metabolites using TMS derivatization pooled according to conditions of $30{ }^{\circ} \mathrm{C}, 37^{\circ} \mathrm{C}$ and $30{ }^{\circ} \mathrm{C}$ with agitation. A.A represents amino acid, F.A represents fatty acid, TCA represents tricarboxylic acid, and O.A represents organic acid.

\begin{tabular}{|c|c|c|c|c|c|}
\hline $30{ }^{\circ} \mathrm{C}$ & Groups & $37^{\circ} \mathrm{C}$ & Groups & $30{ }^{\circ} \mathrm{C}$ agitated & Groups \\
\hline Alanine & A.A & Proline & A.A & Phenylalanine & A.A \\
\hline Leucine & A.A & Leucine & A.A & Leucine & A.A \\
\hline Norleucine & A.A & Phenylalanine & A.A & Serine & A.A \\
\hline Phenylalanine & A.A & 1,3-Propanediol & Alcohol & Acetyl-L-Lysine & A.A \\
\hline Threonine & A.A & 1,2-Butanediol & Alcohol & Aspartic acid & A.A \\
\hline Glycine & A.A & Propanol & Alcohol & Threonine & A.A \\
\hline Ethanol & Alcohol & Decanoic acid & F.A & Glutamine & A.A \\
\hline Propanol & Alcohol & Picolinic acid & F.A & Propanol & Alcohol \\
\hline Acetone & Ketone & Steric acid & F.A & 2-Propanol & Alcohol \\
\hline 2-butanone & Ketone & Hexanoic acid & F.A & 1,2-Benzenediol & Alcohol \\
\hline 2-heptanone & Ketone & Hexadecanoic acid & F.A & Isoleucine & Alcohol \\
\hline Citric acid & TCA & Acetone & Ketone & 1,2-Octanediol & Alcohol \\
\hline Fumaric acid & TCA & 2-Pentanone & Ketone & Cadaverine & Biogenic \\
\hline Acetic acid & O.A & Oxalacetic acid & TCA & Palmitic acid & F.A \\
\hline $\begin{array}{l}\text { Aminobutyric } \\
\text { acid }\end{array}$ & O.A & Lactate & O.A & Hexadecanoic acid & F.A \\
\hline Hexanoic acid & O.A & $\begin{array}{l}\text { 3-Hydroxybutyric } \\
\text { acid }\end{array}$ & O.A & $\begin{array}{l}2,4- \\
\text { Dihydroxyacetophenone }\end{array}$ & Ketone \\
\hline Lactate & O.A & Mercaptoacetic acid & O.A & Heptadecane & Ketone \\
\hline $\begin{array}{l}\text { Mercaptoacetic } \\
\text { acid } \\
\text { Pentadecanoic }\end{array}$ & O.A & Acetic acid & O.A & 2-Pentanone & Ketone \\
\hline acid & O.A & Butanoic acid & O.A & Ethanone & Ketone \\
\hline Propanoic acid & O.A & Octadecanoic acid & O.A & Succinic acid & TCA \\
\hline Glycerin & Polyol & Pentadecanoic acid & O.A & Malic acid & TCA \\
\hline Glycerol & Polyol & $\begin{array}{l}\text { Hexanoic acid } \\
\text { 3-Hydroxypyruvic }\end{array}$ & O.A & Lactate & O.A \\
\hline Uracil & Pyrimidine & acid & O.A & 2-Propenoic acid & O.A \\
\hline Mannose & Sugar & Ascorbic acid & O.A & Mercaptoacetic acid & O.A \\
\hline Ribose & Sugar & Acetic acid & O.A & 3-Hydroxypyruvic acid & O.A \\
\hline Xylose & Sugar & Glycerin & Polyol & Ascorbic acid & O.A \\
\hline Ethanethiol & Sulfur & Glycerol & Polyol & Acetic acid & O.A \\
\hline Glyceraldehyde & & Ribitol & Polyol & Butanoic acid & O.A \\
\hline & & Methanethiol & Sulfurs & Phthalic acid & O.A \\
\hline & & & & 2-Hydroxyisophthalic acid & O.A \\
\hline & & & & Phosphoric acid & O.A \\
\hline & & & & Phenylacetic acid & O.A \\
\hline & & & & Benzoic acid & O.A \\
\hline & & & & Mandelic acid & O.A \\
\hline & & & & Oxalic acid & O.A \\
\hline & & & & Malonic acid & O.A \\
\hline & & & & Hexanoic acid & O.A \\
\hline & & & & 2-Butenedioic acid & O.A \\
\hline & & & & Aminomalonic acid & O.A \\
\hline & & & & Pentanoic acid & O.A \\
\hline
\end{tabular}


Figure 1. Metabolic profiles of L. lactis under growth conditions without agitation at $30^{\circ} \mathrm{C}$, and $37{ }^{\circ} \mathrm{C}$, and $30{ }^{\circ} \mathrm{C}$ with agitation. Profiles were obtained using TMS derivatization and measured by GC-MS.

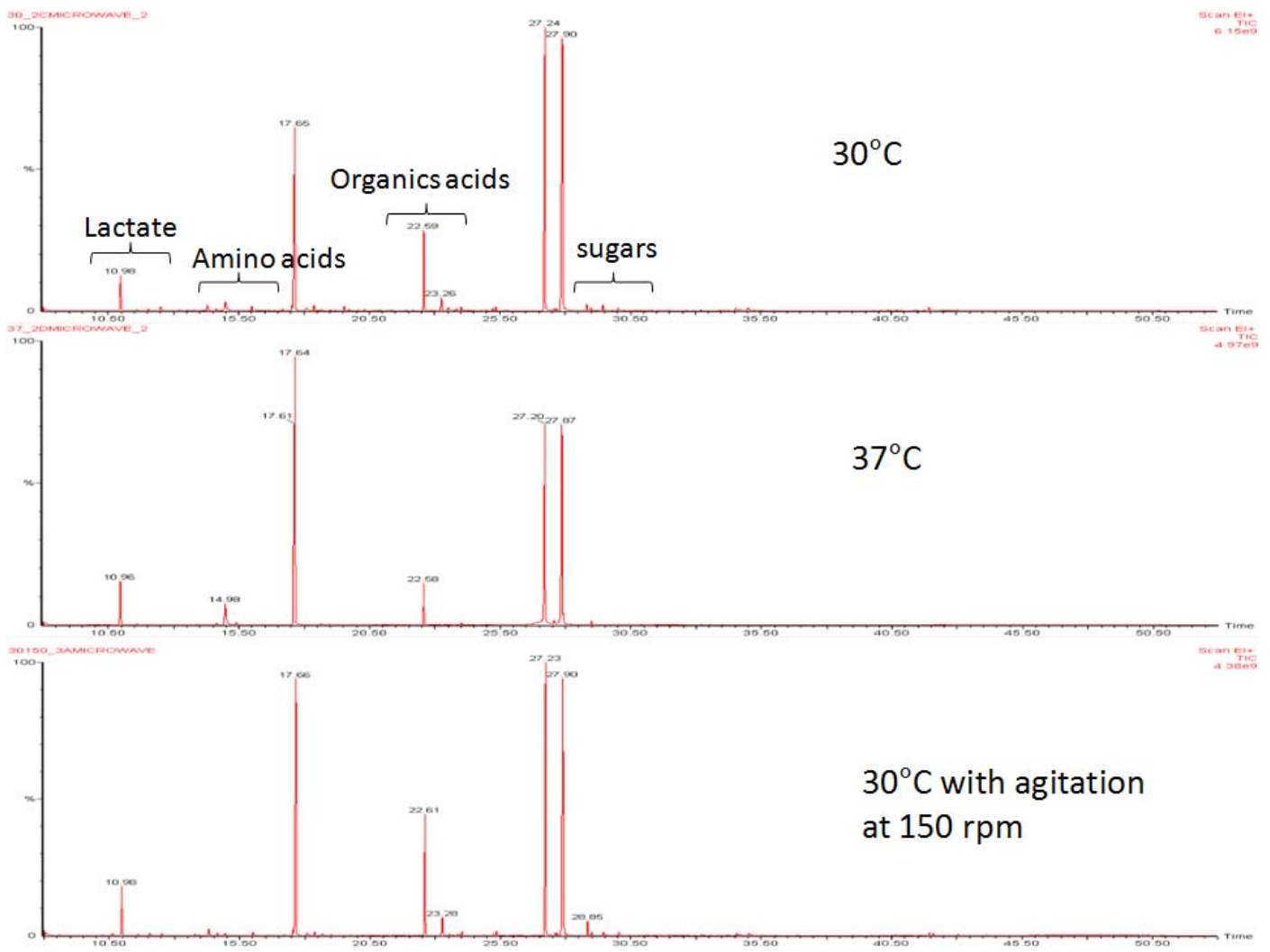

Figure 2. PLS-DA derived loading plot analysis of metabolites profiles. PLS-DA analysis with temperature as the y variable was used to identify the metabolites that distinguished L. lactis grown in non-agitated condition of $30{ }^{\circ} \mathrm{C}$ and $37{ }^{\circ} \mathrm{C}$. Compounds marked red indicate metabolites with variable importance for projection (VIP) values exceeding 1.

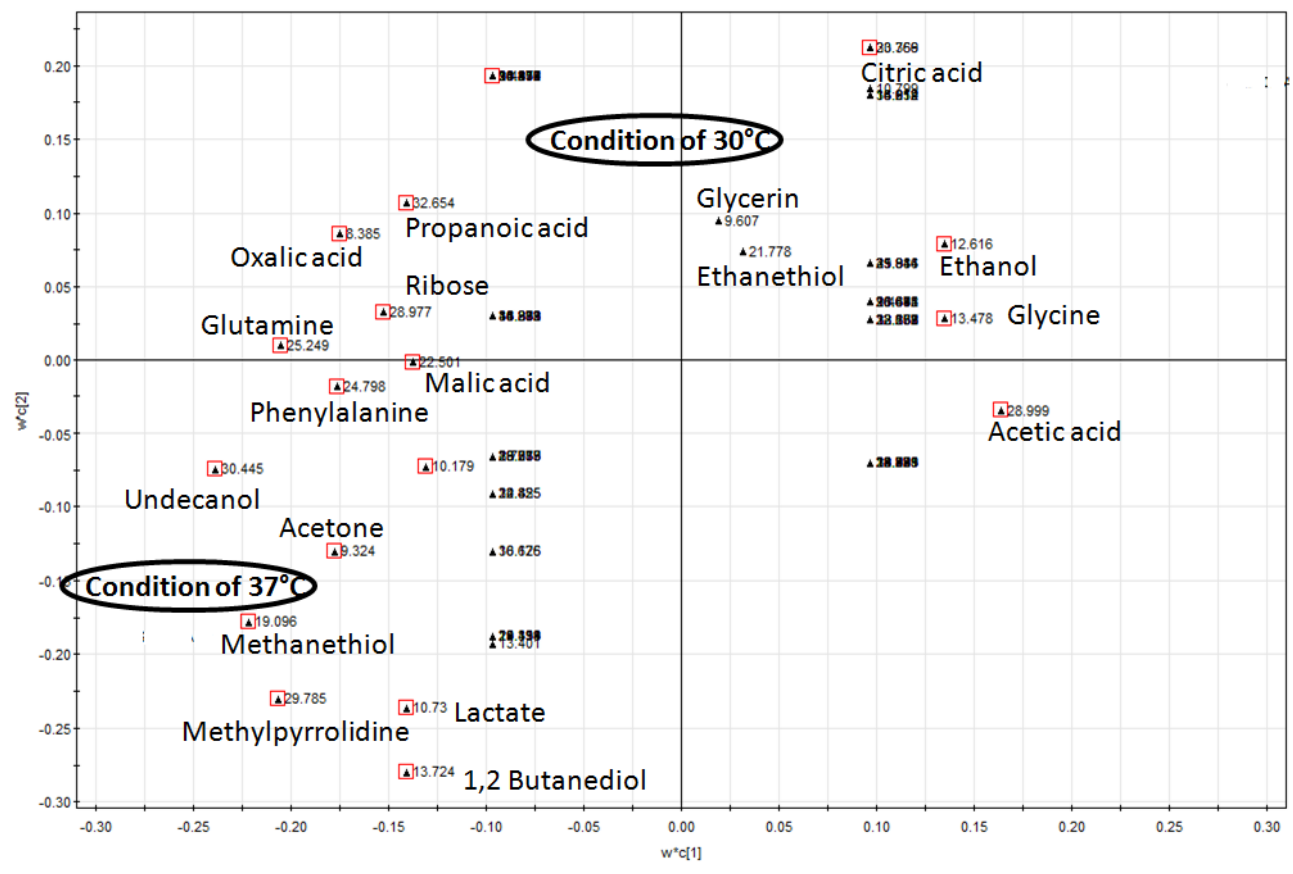

$R 2 \times[1]=0.133944 \quad R 2 \times[2]=0.0828099$ 


\subsection{Changes of Amino Acids and Fermentation End Products}

Multivariate analysis was performed by hierarchical clustering analysis (HCA) to examine the variations of the amino acids and fermentation end products under stresses of temperature and agitation. As shown in Figure 3, some of the aspartate family (aspartate, glutamine, glycine, serine and threonine) and shikimate-derived amino acids (phenylalanine and tyrosine) are more abundant (red) under the $30{ }^{\circ} \mathrm{C}$ agitated condition. In general, aspartate is the precursor to several amino acids including threonine, and isoleucine, while the shikimate pathway via chorismate is essential for the aromatic compounds biosynthesis. Furthermore, greater abundance of phenylalanine and tyrosine particularly under agitated condition suggested a role of phosphoenolpyruvate and erythrose-4phosphate in the activation of shikimate pathway for the production of these aromatic amino acids.

Figure 3. Double hierarchical clustering analysis of detected amino acids from non-agitated conditions of $30{ }^{\circ} \mathrm{C}$ and $37{ }^{\circ} \mathrm{C}$, and $30{ }^{\circ} \mathrm{C}$ with agitation. Red color indicates relatively high abundance; green represents a relatively low abundance.

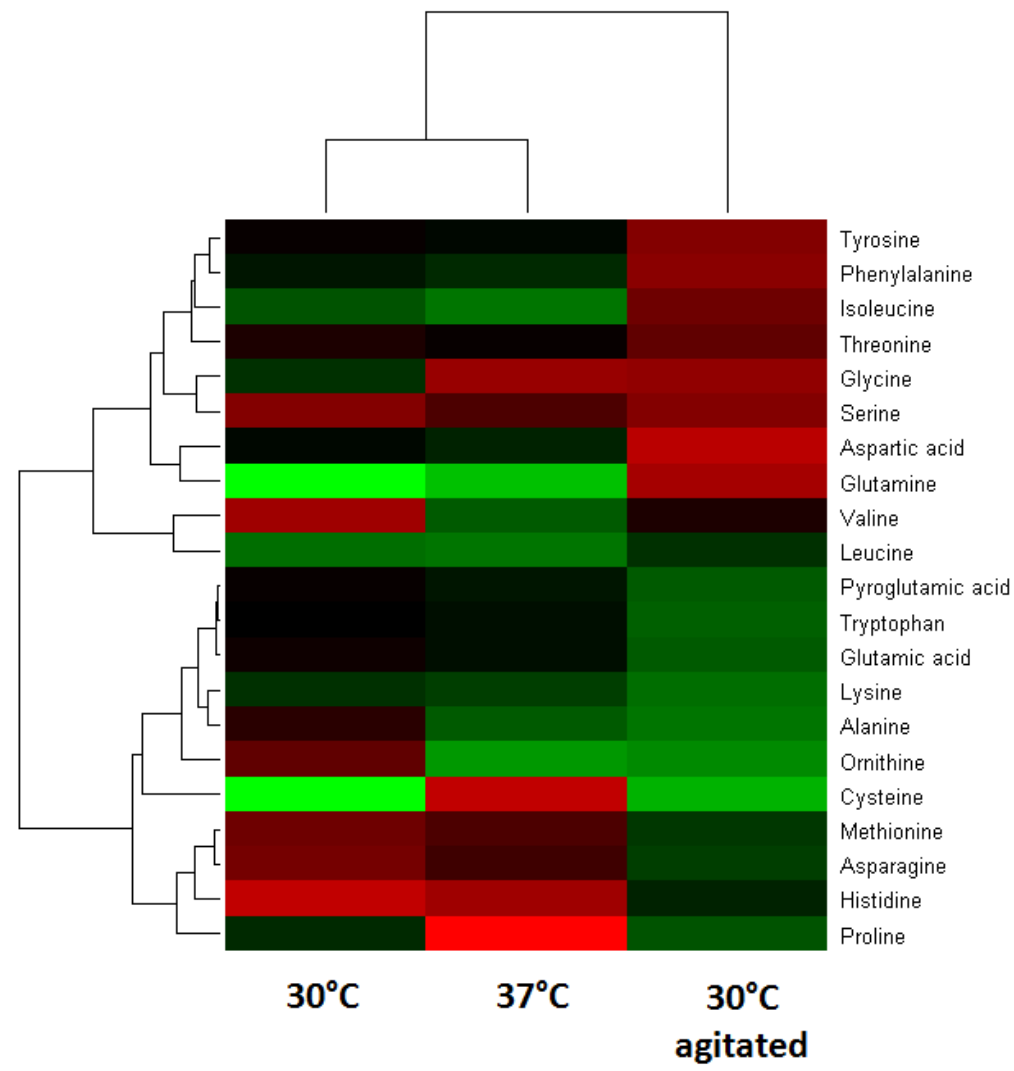

Under non-agitated conditions, ornithine was more abundant at $30{ }^{\circ} \mathrm{C}$, compared with cysteine at $37^{\circ} \mathrm{C}$. The relationship between larger amounts of ornithine and the fermentation end products ethanol and acetate are likely to be associated with carbon limitation. During exponential phase that lasted 5 to $6 \mathrm{~h}$ (data not shown), when the carbon source started to exhaust, there might be a shift toward mixed-acid fermentation. The modification of pyruvate metabolism via pyruvate dehydrogenase activity could explain the production of ethanol and acetate. Furthermore, it was suggested that ornithine is a result from arginine catabolism via the arginine deiminase (ADI) pathway [14]. ADI is responsible for the conversion of arginine into ornithine via citrulline. The activation of ADI was 
influenced by the carbon starvation and changes of $\mathrm{pH}$. In general, ornithine is not a constituent of casein, thus the presence of ornithine indicates the activation of specific enzyme or pathway to generate the amino acid.

A greater amount of cysteine at $37{ }^{\circ} \mathrm{C}$ is consistent to that reported effects at elevated temperature, which affect the incubation time, viable bacterial counts and $\mathrm{pH}$ changes [15]. Cysteine plays important roles in protein folding, assembly and stability via the formation of disulfide bonds [16]. The greater amount of lactate at $37{ }^{\circ} \mathrm{C}$ suggested a metabolic shift to lactate by pyruvate during carbon limitation. This was indicated by little changes in alanine, which is produced from pyruvate that also influences the production of glycine and serine.

\subsection{Changes of Amino Acids in Response to Temperature and Agitation}

The HCA in Figure 3 indicated that there are several clusters (horizontal dendrogram) of amino acids changes with two big clusters, larger one (upper cluster) divided into three groups. The lower clusters that grouped serine, glycine, aspartate, tyrosine, phenylalanine and isoleucine and threonine and glutamine indicated that specific pathways were more activated. As shown in Figure 4, the changes of amino acids that were observed at $30{ }^{\circ} \mathrm{C}$ with agitation indicated role of 3-phosphoglycerate (3PG) and phosphoenol pyruvate (PEP). The larger amounts of valine and leucine suggest the presence of more pyruvate during agitation stresses while the larger amount of isoleucine would require more threonine via 2-oxobutanoate which is derived from oxaloacetate (OAA). Observation of amino acids changes at $30{ }^{\circ} \mathrm{C}$ and $37^{\circ} \mathrm{C}$ suggested role of ribose-5-phosphate (R5P) and oxaloacetate (OAA) due to changes of histidine and asparagine, methionine, and threonine.

Figure 4. Schematic representation of precursor relationship between amino acids and the central carbon metabolism. R5P represents ribose -5-phosphate, 3PG represents 3-phosphoglycerate and PEP represents phosphoenol pyruvate. OAA represents oxaloacetate and AKG represents 2-oxoglutarate while ADI represents the arginine deiminase.

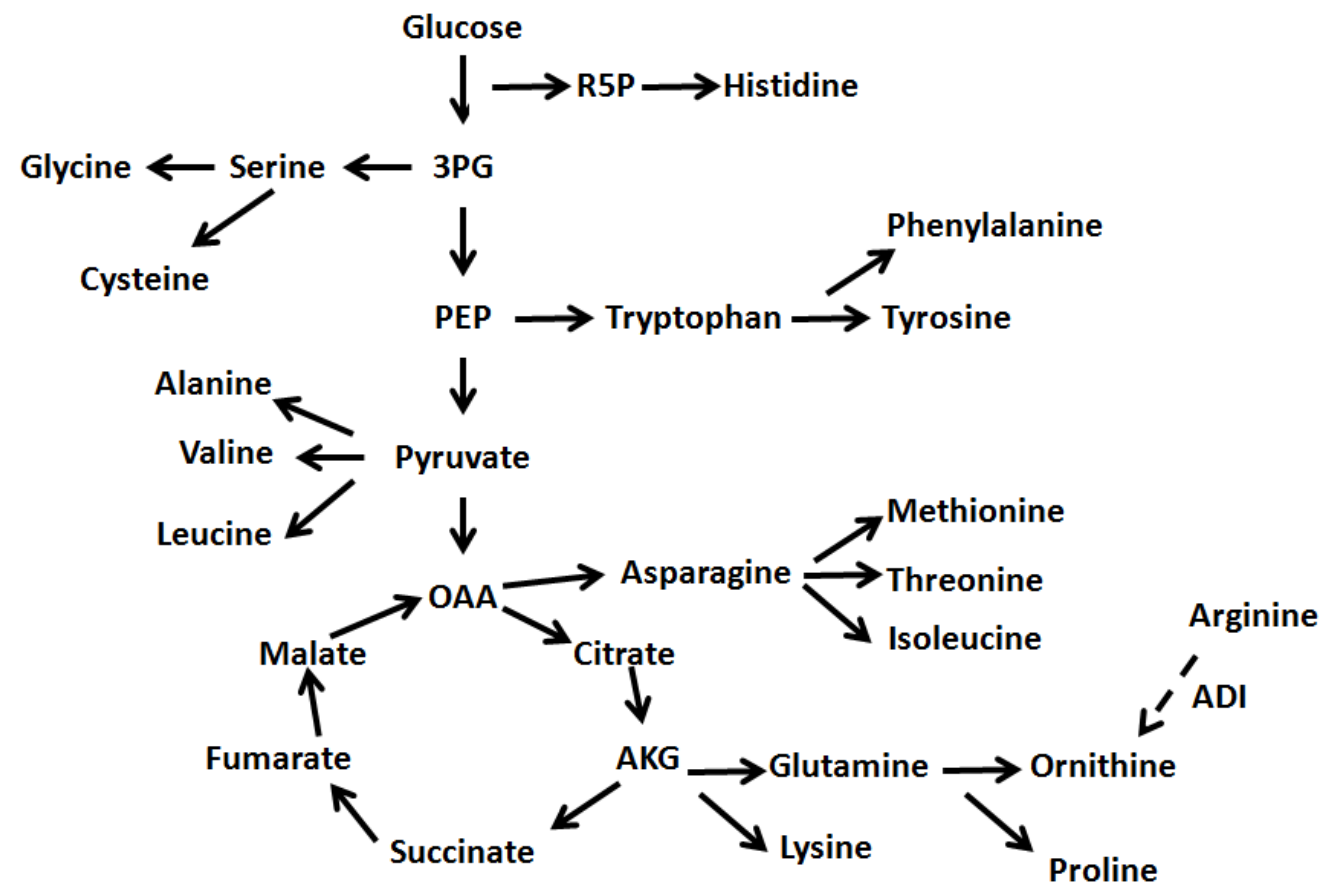




\subsection{Comparison of Metabolites Detected Using TMS, MCF and HS Analysis}

TMS is routinely employed in gas chromatography (GC) to increase the chemical volatility and stability of organic metabolites containing active hydrogen [17]. The derivatization is based on the methoximation and silylation enabling the detection of a wide range of metabolite groups, including sugar derivatives, organic acids, fatty acids and amino acids (Table 1). However, the procedure usually requires a long heating treatment and must be carried out under anhydrous conditions [12,13]. The use of microwave- assistance (MA) in TMS derivatization procedure of MSTFA and BSTFA has significantly increased the detection of metabolites while shortening the heating period of methoximation and silylation steps $[13,18,19]$. The use of microwave irradiation for methoximation and silylation prior to GC-MS analysis has been widely used in biological samples including environmental analysis, herbicides and industrial related processes [19-25]. In this study, MSTFA which works well with microwave application was used instead of other TMS reagent.

The use of MCF derivatization in GC-MS was first introduced by Husek et al. [26]. The derivatization does not require special sample preparation or multiple reaction steps or heating treatment [27]. The alkyl chloroformates based derivatization favors detection of carboxyl group $(-\mathrm{COOH})$ containing metabolites. As shown in Table 2, detected metabolites are mainly amino acids and organic acids, including metabolites from the citrate cycle and fatty acids.

Table 2. Detected metabolites using MCF derivatization are pooled within groups. Metabolites in bold (italic) represent metabolites that are also detected using TMS derivatization.

\begin{tabular}{lll}
\hline Organic acids & Amino acids & Fatty acid \\
\hline 2-aminobutyric acid & Alanine & Caprylate \\
4-aminobutyric acid & Asparagine & 10,12-Otacedecadienoate \\
Caproic acid & Aspartic acid & \\
Citraconate & Cysteine & \\
Fumaric acids & Glutamic acid & \\
Glutaric acid & Glycine & \\
Glyceric acid & Histidine & \\
Hydroxybenzoate & Isoleucine & \\
Itaconic acid & Leucine & \\
Lactate & Lysine & \\
Malic acid & Methionine & \\
Malonic acid & Ornithine & \\
Nicotinic acid & Proline & \\
Oxalacetic acid & Threonine & \\
Oxalic acid & Tryptophan & \\
Succinic acid & Valine & \\
\hline
\end{tabular}

Comparison of groups of metabolite detected by TMS and MCF (Table 3) indicated that MCF derivatization is best suited for targeted profiling. TMS on the other hand detects a wider range of chemical groups making it as a preferable derivatization method for metabolic profiling experiments. 
Table 3. Summary of group of metabolites detected by TMS and MCF.

\begin{tabular}{lcc}
\hline Groups of metabolites & TMS & MCF \\
\hline Alcohols & Yes & No \\
Aldehydes & Yes & No \\
Amines & Yes & No \\
Amino acids & Yes & Yes \\
Fatty acids & Yes & Yes \\
Ketones & Yes & No \\
Organic acids & Yes & Yes \\
Sugars & Yes & No \\
\hline
\end{tabular}

\subsection{Headspace (HS) Analysis}

Direct analysis using dynamic headspace (HS) coupled to GC-MS was carried out to detect any volatile metabolites that react less with the derivatization reagents. Branched chain methyl aldehydes (2-methylbutanal, 3-methylbutanal and 2-methylpropanal) that are commonly produced by L. lactis during cheese manufacturing $[9,28,29]$ were successfully detected, including pentanal and sulfur-based compounds (Figure 5). In brief, aldehydes are the most abundantly produced metabolites by L. lactis, especially in the cheese manufacturing process [28,29]. The production of the branched-chain methyl aldehydes was associated with the lower amounts of branched-chain amino acids of leucine, isoleucine and valine, while pentanal was likely from degradation of unsaturated fatty acids [28]. No production of 2-methylbutanal is observed under the agitation condition, although a high isoleucine response was detected under those conditions.

Figure 5. Bar chart representing the relative abundances of detected branched-chain aldehydes using dynamic headspace (HS) coupled to GC-MS according to the three conditions. Condition with agitation showed less production of branched chain aldehydes. HS_30 represents condition of $30{ }^{\circ} \mathrm{C}, \mathrm{HS} \_37$ represents condition of $37^{\circ} \mathrm{C}$ and HS_30150 represents condition with agitation $(150 \mathrm{rpm})$.

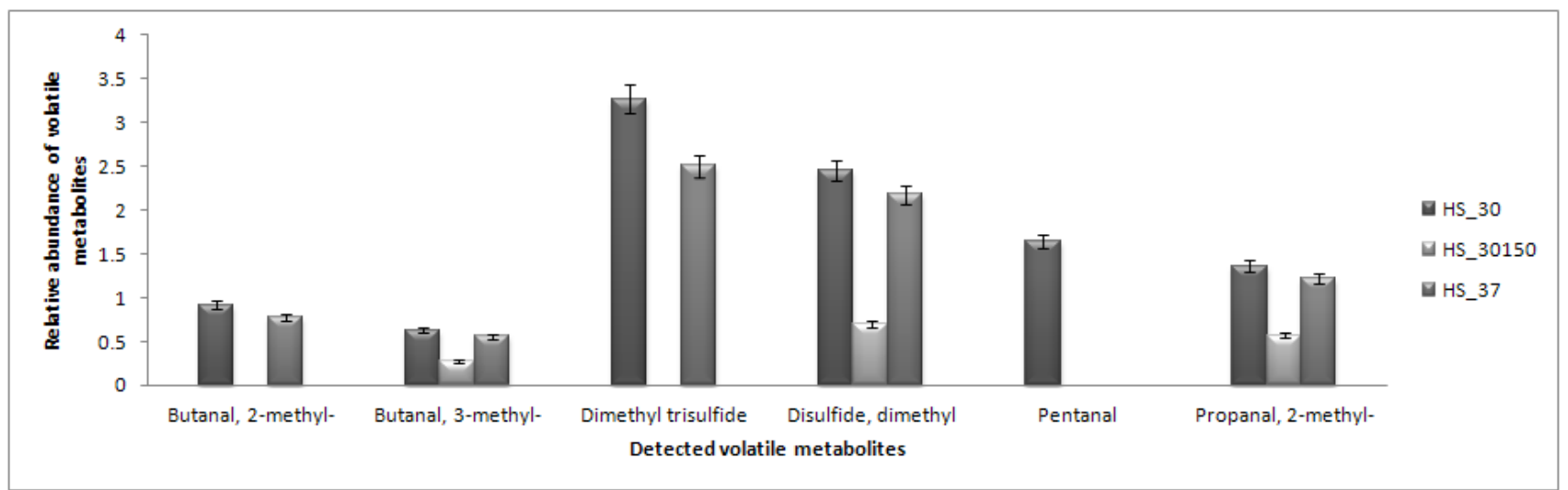

\subsection{Partial Least Square Discriminant Analysis}

Discriminant analysis using supervised PLS-DA for samples derivatised using TMS (Figure 6A) and MCF (Figure 6B) indicated clear separation between the $30^{\circ} \mathrm{C}, 30^{\circ} \mathrm{C}$ with agitation and $37^{\circ} \mathrm{C}$. 
As MCF derivatization favours the detection of amino acids, the PLS-DA score plot (Figure 6B) is likely to be influenced by the amino acids response. This is supported by the hierarchical clustering analysis (HCA) on the particular metabolites that revealed amino acids to influence the discrimination (Figure 6C).

Figure 6. (a) PLS-DA score plots by the combination of PC1 and PC 2 of TMS derivatised replicates. The ellipses represent confidences of $95 \%$ in the Hotelling T2 tests. 30 represents condition of $30{ }^{\circ} \mathrm{C}, 37$ represents condition of $37{ }^{\circ} \mathrm{C}, 30150$ represents condition with agitation (150 rpm). (b) PLS-DA score plots by the combination of PC1 and PC2 of MCF derivatised replicates. The ellipses represent confidences of $95 \%$ in the Hotelling T2 tests. 30 represents condition of $30{ }^{\circ} \mathrm{C}, 37$ represents condition of $37{ }^{\circ} \mathrm{C}, 30150$ represents condition with agitation (150 rpm). (c) Hierarchical clustering analysis of MCF derivatised metabolites according to conditions. Red represents condition of $30{ }^{\circ} \mathrm{C}$, blue represents the condition of agitation and black represents the condition of $37^{\circ} \mathrm{C}$.

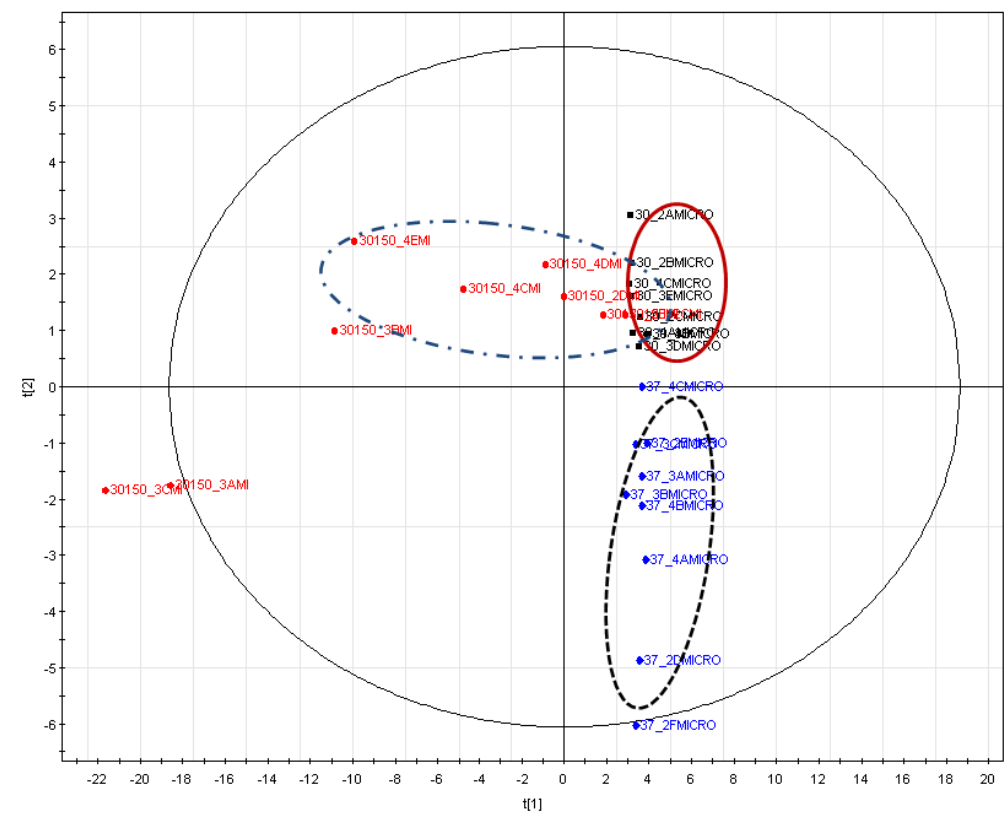

(a)

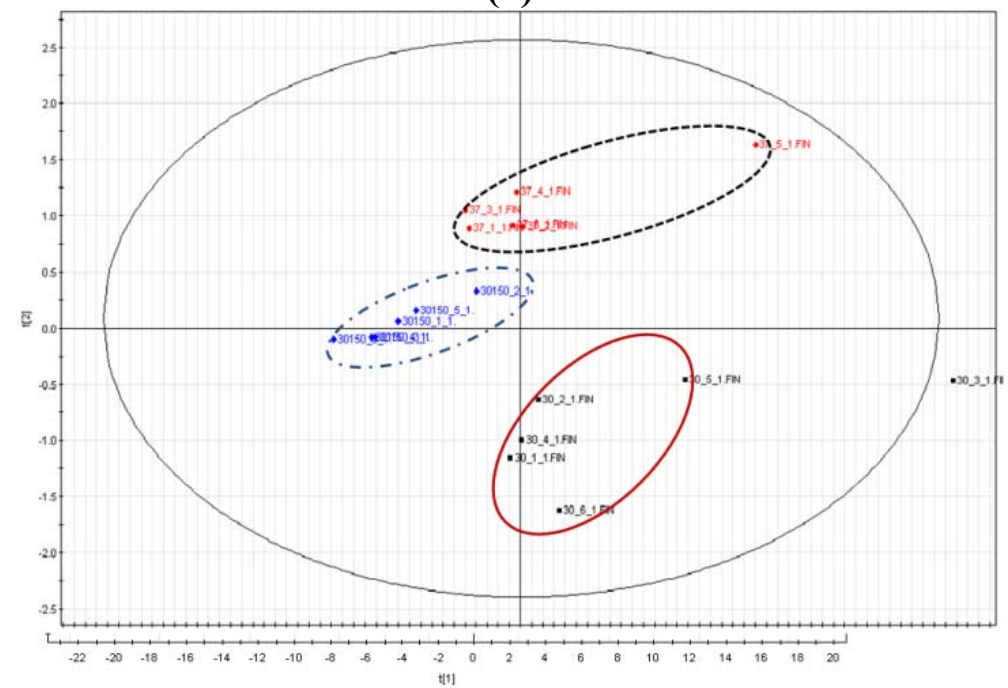

(b) 
Figure 6. Cont.

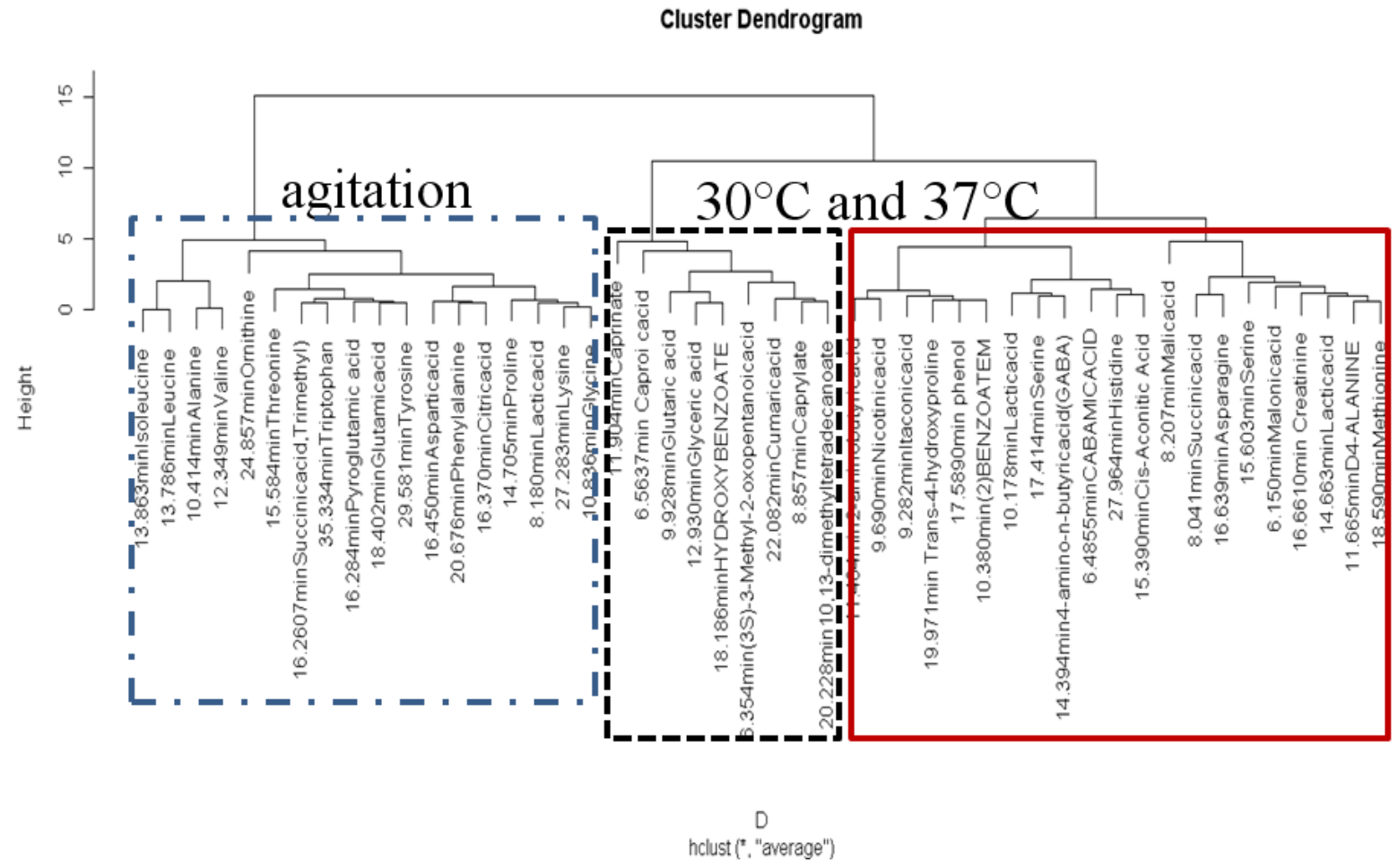

(c)

\section{Experimental}

\subsection{Chemicals}

All chemical reagents used were of analytical grade and purchased from different suppliers (Sigma, Merck, and Burker Corporation). Methylchloroformate (MCF), $N$-methyl- $N$-trimethysilyl trifluoroacetamide (MSTFA) and methoxyamine hydrochloride were obtained from Sigma (St. Louis, MO, USA). Pyridine was purchased from Merck (Whitehouse Station, USA).

\subsection{Microbial Cultivation}

The plasmid-cured L. lactis MG 1363 culture was kindly provided by K. Leenhouts (University of Groningen, Groningen, The Netherlands) and Raha Abd. Rahim (Universiti Putra Malaysia, Serdang, Malaysia). Fermentations were performed under aerobic conditions $\left(\mathrm{O}_{2}\right.$ environment $)$ at $30{ }^{\circ} \mathrm{C}$ and $37{ }^{\circ} \mathrm{C}$ without agitation and $30{ }^{\circ} \mathrm{C}$ with agitation at $150 \mathrm{rpm}$. The M17 medium used as cultivation medium, (Oxoid Limited, Hampshire, UK) as described by Terzaghi and Sandine [30] contained (in $\mathrm{g} \mathrm{L}^{-1}$ ): ascorbic acid (0.5), $\mathrm{MgSO}_{4}(0.25)$, disodium glycerophosphate (19), tryptone (5), soytone (5), beef extract (5) and yeast extract (2.5). Glucose (0.5\%) was added as the carbon source. Samples were collected during the exponential growth (5-6 hours) with $\mathrm{OD}_{600 \mathrm{~nm}}$ of 1.0 .

\subsection{Growth Estimation}

Optical density (OD) at $600 \mathrm{~nm}$ was used to provide a measure biomass and constructing growth curve for each of cultivation under the specific conditions. 


\subsection{Extraction of Extracellular Samples}

Approximately $15 \mathrm{~mL}$ of fermented culture medium was taken and filtered using a cellulose acetate membrane filter $(0.2 \mu \mathrm{m}$ pore size $)$ to remove the microbial cells. Filtered culture medium was then separated into $1 \mathrm{~mL}$ aliquots $(\mathrm{n}=5)$ followed by the addition of $\mathrm{dH}_{2} \mathrm{O}(10 \mathrm{~mL})$ and internal standard ( $0.2 \mu \mathrm{mol}$ of $10 \mathrm{mmol}$ solution of $2,3,3,3-\mathrm{d} 4 \mathrm{D}, \mathrm{L}$-alanine) to each of the samples. Samples were then freeze dried under low temperature $\left(-56^{\circ} \mathrm{C}\right)$ and stored at $-20{ }^{\circ} \mathrm{C}$. Un-inoculated M17 broth (WT) was also prepared as control.

\subsection{Sample Derivatization Using TMS}

The TMS derivatization method was based on the optimized protocol described by Villas-Boas et al. [25] and Rossner et al. [31] Briefly, freeze dried samples were resuspended in methoxyamine hydrochloride solution in pyridine $(80 \mu \mathrm{L}, 2 \mathrm{~g} / 100 \mathrm{~mL})$, followed by incubation in a domestic microwave (Panasonic NN-K544WF) with multimode irradiation set to $500 \mathrm{~W}$ and $50 \%$ of exit power for $2.48 \mathrm{~min}$. MSTFA was then added (approximately $80 \mu \mathrm{L}$ ), followed by incubation in the domestic microwave for $3 \mathrm{~min}$, under same conditions as previously mentioned. The final mixed incubation sample was then transferred to a GC-MS vial and analyzed by GC-MS.

\subsection{Sample Derivatization Using $M C F$}

The MCF derivatization method was based on protocol described by Smart et al. [27] and Villas-Boas [25]. Briefly, freeze dried samples were resuspended in $\mathrm{NaOH}$ (1M), followed by addition of methanol, pyridine, MCF and chloroform and sodium bicarbonate. The upper aqueous layer in the sample was discarded, and a small portion of anhydrous sodium sulphate was added to dry the remaining reagents. Finally, the dried solution was transferred into a GC-MS vial and analyzed by GC-MS.

\subsection{Sample Preparation for Headspace Analysis (HS)}

Briefly, freeze dried samples were resuspended in $200 \mu \mathrm{L}$ of $\mathrm{dH}_{2} \mathrm{O}$ and homogenized under temperature of $40{ }^{\circ} \mathrm{C}$ before being loaded in headspace apparatus and analyzed using GC-MS.

\subsection{GC-MS Parameter for Samples Prepared by TMS}

The GC-MS parameter used was optimized based on Villas-Boas et al. [25] and Rossner et al. [31]. GC-MS analysis was performed using the GC-MS Perkin Elmer Turbo Mass Clarus 600 coupled to a quadruple mass selective detector on electron ionization (EI) operated at $70 \mathrm{eV}$. An aliquot of approximately $1-\mu \mathrm{L}$ was injected into an Elite-5MS capillary column coated with $5 \%$ diphenyl crosslinked and $95 \%$ dimethylpolysiloxane $(30 \mathrm{~m} \times 0.25 \mathrm{~mm}$ i.d. $\times 0.25 \mu \mathrm{m}$ thickness $)$ in split mode (50:1). The injection temperature was set to $250^{\circ} \mathrm{C}$, and the ion source temperature was adjusted to $200{ }^{\circ} \mathrm{C}$. The GC method was set from $70{ }^{\circ} \mathrm{C}$ to $300{ }^{\circ} \mathrm{C}$ with helium gas flow constantly at $1.1 \mathrm{~min}^{-1}$. The measurements were made in the full scan mode $(\mathrm{m} / \mathrm{z} 45-600)$. 


\subsection{GC-MS Parameters for Samples Prepared by $M C F$}

The GC-MS parameter used was described by Smart et al. [27] and Villas-Boas et al. [25]. GC-MS analysis was performed using Agilent GC-MS coupled to a quadruple mass selective detector on electron ionization (EI) operated at $70 \mathrm{eV}$. An aliquot of approximately $1-\mu \mathrm{L}$ was injected into $\mathrm{J} \& \mathrm{~W}$ 1701 column $(30 \mathrm{~m} \times 250 \mathrm{~mm}$ i.d. $\times 0.15 \mathrm{~mm})($ Folsom, CA $)$. The injection temperature was set to $250{ }^{\circ} \mathrm{C}$, and the ion source temperature was adjusted to $200{ }^{\circ} \mathrm{C}$. The $\mathrm{GC}$ method was set from $45^{\circ} \mathrm{C}$ to $280{ }^{\circ} \mathrm{C}$ with helium gas flow constantly at $1.0 \mathrm{~min}^{-1}$. The measurements were made in the scan mode of $38-650 \mathrm{~m} / \mathrm{z}$ at $1.47 \mathrm{scan}$ per sec.

\subsection{GC-MS Parameters for Headspace Analysis (HS)}

A Perkin Elmer TurboMatrix Headspace Sampler 40XL connected to a GC-MS Perkin Elmer Turbo Mass Clarus 600 was used for volatile compounds analysis. A minimal of three duplicates were subjected to helium purge and concentrated in a Tenax trap, kept at $40{ }^{\circ} \mathrm{C}$. Line temperature was adjusted to $180{ }^{\circ} \mathrm{C}$, while helium flow was set at $40 \mathrm{~mL} / \mathrm{min}$. Sample temperature was $80{ }^{\circ} \mathrm{C}$, with dry purge time was $1 \mathrm{~min}$ and desorbed temperature was $200{ }^{\circ} \mathrm{C}$. Desorbed time was $1 \mathrm{~min}$, and injection port temperature was set to $200{ }^{\circ} \mathrm{C}$. GC-MS analysis was performed using electron ionization (EI) operated at $70 \mathrm{eV}$. An aliquot of approximately $1 \mu \mathrm{L}$ was injected into an Elite-5MS capillary column coated with $5 \%$ diphenyl crosslinked and $95 \%$ dimethylpolysiloxane $(30 \mathrm{~m} \times 0.25 \mathrm{~mm}$ i.d. $\times 0.25 \mu \mathrm{m}$ thickness) in split mode (50:1). The injection temperature was set to $250{ }^{\circ} \mathrm{C}$, and the ion source temperature was adjusted to $200{ }^{\circ} \mathrm{C}$. The $\mathrm{GC}$ method was set from $45{ }^{\circ} \mathrm{C}$ to $220{ }^{\circ} \mathrm{C}$ with helium gas flow constantly at $1.0 \mathrm{~mL} \mathrm{~min}^{-1}$. The measurements were made in the scan mode of $\mathrm{m} / \mathrm{z} 33-220$.

\subsection{Data Analysis and Validation}

The general approach used for data analysis and validation was performed according to Smart et al. [27] and Villas-Boas et al. [25]. In summary, detected metabolites were identified using in-house TMS and MCF MS library of derivatised pure standard developed by Villas-Boas. For peaks that have not been identified was identified using NIST mass spectral database library (NIST 2008) with cut-off similarity of $90 \%$. The value of height of the peak was used to represent the detected metabolites. The values were firstly normalized by total sum of GC height and internal standard followed by $\log$ transformed. One-way Analysis of Variance (ANOVA) was used to statistically validate the values followed by comparison using Fisher's least significant difference (LSD) method with significance levels of $P<0.05, P<0.01$ and $P<0.001$ [32]. Visualization of the clean, validated data was then carried out using Principal Component Analysis (PCA) and PLS-DA of Simca-P+ version 12.0 (Umetrics AB, Ume, Sweden) for group classification and discrimination analysis with $\mathrm{Q}^{2}$ value $>50 \%$. The heatmap with hierarchical clustering analysis was performed using $\mathrm{R}$ script (http://www.r-project.org) with the ward method.

\section{Conclusions}

GC-MS and HS analysis on the metabolites produced by L. lactis in response to temperature and agitation contribute to the understanding of metabolic changes during environmental stresses. 
Meanwhile, the use of TMS derivatization provides a wider range of metabolites detection compared to MCF derivatization which specifically targets amino acids. The PLS-DA derived analyses indicate a strong relationship between fermentation end products of lactate, ethanol, acetate and amino acid changes according to temperature. Finally, these specific responses can serve as optimization factors useful for dairy food production which uses L. lactis as starter culture.

\section{Acknowledgments}

The research was funded by Ministry of Science and Technology (MOSTI) under the genomics and molecular biology initiative grant research (UKM-MGI-NBD000812007) and Research University Grant (GUP-2011-268). We thank Raha Abd. Rahim from Universiti Putra Malaysia for providing L. lactis strains and, Silas Villas-Boas, Kathleen F. Smart, Raphael B. M. Aggio, Jeremy R. Van Houtte, Morgan Han, and Hans from University of Auckland, New Zealand for the assistance and technical support. We also would like to thank Michael Meyrick Burrell from University of Sheffield, United Kingdom for his helpful comments and intensive English editing of the manuscript.

\section{Conflict of Interest}

The authors declare no conflict of interest.

\section{References and Notes}

1. Losiane, E.G.; Sylvain, M. Bacteriophages of lactic acid bacteria and their impact on milk fermentations. Microb. Cell Fact. 2011, 10, S20.

2. Bermúdez-Humarán, L.G.; Kharrat, P.; Chatel, J.-M.; Langella, P. Lactococci and lactobacilli as muscosal delivery vectors for therapeutic proteins and DNA vaccines. Microb. Cell Fact. 2011, $10, \mathrm{~S} 4$.

3. Mireille, Y.; Chistophe, G.; Emilie, C.; Gaelle, B.; Veronique, M. The initial efficiency of the proteolytic systems of Lactococcus lactis strains determines their responses to a cheese environment. Int. Dairy J. 2011, 21, 335-345.

4. Guimont, C. Change of free amino acids in M17 medium after growth of Streptococcus thermophilus and identification of a glutamine transport ATP-binding protein. Int. Dairy J. 2002, 12, 729-736.

5. Amel, T.; Nassra, D.; Maryse, L.; Denis, R.; Gisele, L. Comparative transcriptome analysis of Lactococcus lactis subsp. cremoris strains under conditions simulating Cheddar cheese manufacture. Int. J. Food Microbiol. 2011, 146, 263-275.

6. Ziadi, M.; Bergot, G.; Courtin, P.; Chambellon, E.; Hamdi, M.; Yvon, M. Amino acid catabolism by Lactococcus lactis during milk fermentation. Int. Dairy J. 2010, 20, 5-31.

7. Ayad, E.H.E.; Verheul, A.; de Jong, C.; Wouters, J.T.M.; Smit, G. Flavour forming abilities and amino acid requirements of Lactococcus lactis strains isolated from artisanal and non-dairy origin. Int. Dairy J. 1999, 19, 725-735.

8. Clementine, D.; Emma, R.; Christophe, G.; Pascal, L.; Veronique, M.; Muriel, C.B. Investigation of the adaptation of Lactococcus lactis to isoleucine starvation integrating dynamic transcriptome and proteome information. Microb. Cell Fact. 2011, 10, S18. 
9. Grade, S.; Carbonell, M.; Fernandez-grarcia, E.; Medina, M.; Nunez, M. Volatile compounds in Hispanico Cheese manufactured using a mesophilic starter a thermophilic starter and bacteriocinproducing Lactococcus lactis subps. cremoris INIA415. J. Agric. Food Chem. 2002, 50, 6752-6757.

10. Zhu, D.; Zhou, X.; Jin, Y.Y. Metabolome profiling reveals adaptive evolution of S. Cerevisiae during repeated vacuum fermentations. Metabolomics 2010, 870, 222-232.

11. Villas-Boas, S.G.; Delicado, S.G.; Akesson, M.; Nielsen, J. Simultaneous analysis of amino and nonamino organic acids as methyl chloroformate derivatives using gas chromatography-mass spectrometry. Anal. Biochem. 2003, 322, 134-138.

12. Cheng, W.P.; Yang, X.Y.; Hegeman, A.D.; Gray, W.M.; Cohen, J.D. Microscale analysis of amino acids using gas chromatography-mass spectrometry after methyl chloroformate derivatization. J. Chromatogr. B 2010, 878, 2199-2208.

13. Liebeke, M.; Wunder, A.; Lalk, M. A rapid microwave-assisted derivatization of bacterial metabolome samples for gas chromatography/mass spectrometry analysis. Anal. Biochem. 2009, $401,312-314$.

14. Urancken, G.; Rimaux, T.; Wouters, D.; Leny, F.; De Vuyst, L. The arginine deiminase pathway of L. fermentum IMDO 13010 response to growth under stress conditions of both temperature and salt. Food Microbiol. 2009, 7, 720-727.

15. Dong, X.; Quinn, P.J.; Wang, X. Metabolic engineering of Escherichia coli glutamicum for the production of L- threonine. Biotechnol. Adv. 2011, 29, 11-23.

16. Brice, S.; Patrice, P.; Dusko, S.E.; Pierre, R.; Eric, G. Sulfur amino acid metabolism and its control in L. lactis IL1403. J. Bacteriol. 2005, 11, 3762-3778.

17. Beale, D.J.; Dunn, M.S.; Morrison, P.D.; Porter, N.A.; Marlow, D.R. Characterisation of bulk water samples from copper pipes undergoing microbially influenced corrosion by diagnostic metabolomic profiling. Corros. Sci. 2012, 55, 272-279.

18. Kouremenos, K.A.; Harynuk, J.J.; Winniford, W.L.; Morrison, P.D.; Marriott, P.J. One-pot microwave derivatization of target compounds relevent to metabolomics with comprehensive two-dimensional gas chromatography. J. Chromatogr. B 2010, 878, 1761-1770.

19. Chu, T.Y.; Chang, C.H.; Liao, Y.C.; Chen, Y.C. Microwave-accelerated derivatization processes for the determination of phenolic acids by gas chromatography-mass spectrometry. Talanta 2001, $54,1163-1171$.

20. Ranz, A.; Maier, E.; Motter, H.; Lankmayr, E. Extraction and derivatization of polar herbicides for GC-MS analyses. J. Sep. Sci. 2008, 31, 3021-3029.

21. Bowden, J.A.; Dominic, M.C.; Whitney, L.S.; Diana, C.M.M.; Timothy, J.G.; Richard, A.Y.; Enhanced Analysis of Steroids by Gas Chromatography/Mass Spectrometry using MicrowaveAccelerated Derivatization. Anal. Chem. 2009, 16, 6725-6734.

22. Beale, D.J.; Michael, S.D.; Donavan, M. Application of GC-MS metabolic profiling to "blue-green water" from microbial influenced corrosion in copper pipes. Corros. Sci. 2010, 52, 3140-3145.

23. Sandra, L.S.; Markus, D.; Oliver Kappe, C. Microwave-assisted derivatization procedures for gas chromatography/mass spectrometry analysis. Mol. Divers. 2010, 14, 869-888. 
24. Ruiz-Matute, A.I.; Hernandez-Hernandez, O.; Rodriguez-Sanchez, S.; Sanz, M.L.; Martinez-Castro, I. Derivatization of carbohydrates for GC and GC-MS analyses. J. Chromatogr. B 2011, 879, 1226-1240.

25. Villas-Boas, S.G.; Noel, S.; Lane, G.A.; Attwood, G.; Cookson, A. Extracellular metabolomics: A metabolic footprinting approach to assess fiber degradation in complex media. Anal. Biochem. 2006, 349, 297-305.

26. Husek, P.; Matucha, P.; Vrankova, A.; Simek, P. Simple plasma work-up for a fast chromatographic analysis of homocysteine, cysteine, methionine and aromatic amino acids. J. Chromatogr. B 2003, 789, 311-322.

27. Smart, K.F.; Aggio, R.M.B.; Houtte, J.R.V.; Villas-Boas, S.G. Analytical platform for metabolome analysis of microbial cells using methyl chloroformate derivatization followed by gas chromatography-mass spectrometry. Nat. Protocol 2010, 5, 1709-1729.

28. Yvon, M.; Rijnen, L. Cheese flavour formation by amino acid catabolism. Int. Dairy J. 2001, 11, 185-201.

29. Marilley, L.; Casey, M.G. Flavours of cheese products: Metabolic pathways analytical tool and identification of producing strains. Int. J. Food Microbiol. 2004, 90, 139-159.

30. Terzaghi, B.E.; Sandine, W.E. Improved medium for lactic streptococci and their bacteriophages. App. Microbiol. 1975, 29, 807-813.

31. Roessner, U.; Wagner, C.; Kopka, J.; Trethewey, R.N.; Willmitzer, L. Simultaneous analysis of metabolites in potato tuber by gas chromatography-mass spectrometry. Plant J. 2010, 23, $131-142$.

32. Xia, J.; Psychogios, N.; Young, N.; Wishart, D.S. MetaboAnalyst: A web server for metabolomic data analysis and interpretation. Nucleic Acids Res. 2009, 37, 652-660.

Sample Availability: Samples of the metabolites lists are available from the authors.

(C) 2012 by the authors; licensee MDPI, Basel, Switzerland. This article is an open access article distributed under the terms and conditions of the Creative Commons Attribution license (http://creativecommons.org/licenses/by/3.0/). 